\title{
Another Consequence of Severe Lupus: Invasive Fungal Disease
}
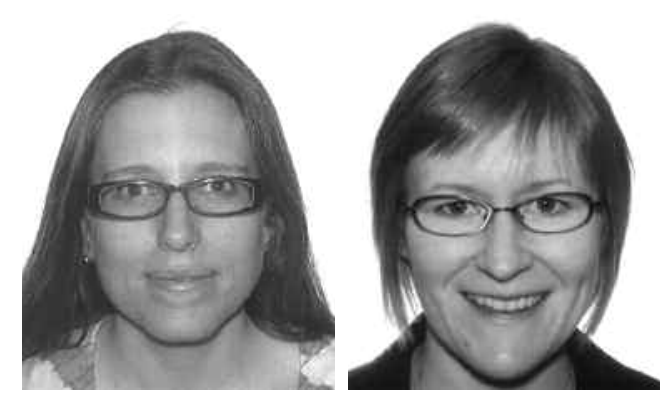

Infections are a substantial cause of morbidity and mortality in patients with systemic lupus erythematosus (SLE), accounting for one-quarter of all deaths ${ }^{1,2}$. The mechanisms for increased susceptibility to infections in patients with SLE are classically attributed to disease- and treatment-related factors. Polymorphisms in the mannose-binding lectin ${ }^{3}$ protein, which helps in host defense by activating the complement cascade, have been reported as a risk factor primarily for bacterial infections in $\mathrm{SLE}^{3}$. Patients with SLE may also have acquired hypogammaglobulinemia (as reviewed by Yong, et $a l^{4}$ ) or hyposplenism, which leads to increased susceptibility to infections with encapsulated organisms. Acquired neutropenia has also been cited as a risk factor for infections in $\mathrm{SLE}^{5}$ and for fungal infections in other populations ${ }^{6}$. Corticosteroids are frequently necessary in the treatment of SLE, resulting in diminished cellular immunity. Immunosuppressant therapy also clearly increases the risk of bacterial infection ${ }^{7}$.

Invasive fungal infections were initially defined in $2002^{8}$, with revisions occurring in 2008 , including a change in terminology to "invasive fungal disease" (IFD) ${ }^{9}$ by the European Organization for Research and Treatment of Cancer/Invasive Fungal Infections Cooperative Group and the National Institute of Allergy and Infectious Diseases Mycoses Study Group (EORTC/MSG) Consensus Group. Proven IFD are subdivided into molds (Aspergillus) and yeasts (Candida species and Cryptococcus) and depend on culture or microscopic analysis from sterile anatomic sites, blood cultures, or cryptococcal antigen in cerebrospinal fluid. Separate criteria exist for defining proven endemic mycoses (Coccidioides species, Histoplasma, and Blastomyces $)^{9}$. The definitions of probable and possible infections are more complex, as described in detail by De Pauw, et $a l^{9}$. Of note, Pneumocystis jirovecii infections are not included in these definitions ${ }^{9}$.

IFD definitions were developed based on systematic reviews of the infection literature in the hematology and oncology fields, and have been applied in clinical trials and epidemiologic studies (including the study by Martínez-Martínez, et al in this issue of The Journal ${ }^{10}$ ). These definitions have not been widely applied to patients with rheumatic conditions although the revised criteria now acknowledge connective tissue disorders as a "host factor" with susceptibility to IFD ${ }^{9}$.

The literature of IFD in SLE consists largely of case reports and case series. The most recent, by Chen, et $a l^{11}$, was a retrospective review of 18 SLE patients with IFD compared to patients with active SLE and with other infectious diseases such as tuberculosis and bacterial infections. Cryptococcus was the most common pathogen in those with IFD and was implicated in 50\% of cases. Frequent sites for infection included the lungs or central nervous system, with disseminated infection also being recognized. Three deaths occurred. Compared to patients with other infections or patients with active SLE, those with IFD were younger, with shorter disease duration. They also had higher doses of steroids and were more likely to have previous antibiotic exposure; however, recent cyclophosphamide use was not a risk factor ${ }^{11}$.

A retrospective review of 2344 SLE patients over 26 years in Taiwan found 15 cases of IFD including 11 (73.7\%) with Cryptococcus, identified primarily in the cerebrospinal fluid $^{12}$. Seven of the patients died from their IFD (15.5\%), with 6 of the deaths due to Cryptococcus, and the overall survival of the remaining patients was also significantly reduced $^{12}$. Given the small number of events, statistical analysis of features related to the infection including disease activity, immunosuppressant medications, corticosteroid doses, and laboratory variables was extremely limited. The authors did note that that active SLE [as measured by the SLE Disease Activity Index ${ }^{13}$ (SLEDAI) > 7] was a potential predisposing factor, as all patients had active disease, and high doses of steroids after diagnosis of IFD were associated with mortality.

See Invasive fungal infections in SLE, page 1814 
Other case series in patients with SLE have reported invasive Candida $s p$. infections ${ }^{14}$ or Aspergillus infections ${ }^{15}$, and highlight corticosteroid medications as possible risk factors for IFD ${ }^{14,15}$. The differing causative organisms among centers are felt to be multifactorial and may be related to differing treatment patterns for SLE or different prevalence of fungi and molds in their particular environment ${ }^{15}$.

In this issue of The Journal Martínez-Martínez, et al present one of the largest series of patients with SLE complicated by IFD in the literature ${ }^{10}$. Over a 7 -year period, 10 of 309 hospitalized SLE patients in Mexico were identified to have IFD. The most commonly reported pathogens were Candida species presenting with disseminated infections. A 70\% mortality rate was seen in the patients with IFD. The study also compares demographic and clinical characteristics of the patients with IFD to 50 hospitalized SLE patients. Those with IFD were more likely to be on hemodialysis, on doses of corticosteroids $>0.5 \mathrm{mg} / \mathrm{kg} / \mathrm{day}(\mathrm{p}<0.001)$, and to have higher disease activity as measured by the SELENA (Safety of Estrogens in Lupus Erythematosus National Assessment)-SLEDAI compared to controls $(14 \pm 9.5$ vs $8 \pm 6$; $p=$ 0.004). Patients with IFD were also taking mycophenolate mofetil in higher numbers compared to their counterpart controls, although the numbers presented in the study are too small to describe definite associations. A limitation of the study that the authors acknowledge is that not all 309 hospitalized patients were actively investigated for IFD, and it is possible some additional cases were missed.

The authors found that C-reactive protein (CRP) levels were higher in subjects with IFD and suggest that a CRP level $>10 \mathrm{mg} / \mathrm{dl}$ could be a useful flag to alert physicians to investigate for IFD ${ }^{10}$. Interestingly, in the study by Chen, et $a l^{11}$ a blunted CRP response was noted in patients with IFD, although the implicated pathogens differed significantly between the 2 studies. We do not feel that CRP is the best marker for IFD as it is not specific enough, and a low level may be falsely reassuring. Although elevated CRP may be a clue to an infection in patients with SLE, the addition of other clinical features should prompt investigation for IFD including unexplained fevers, central nervous system findings, lymphadenopathy, or diffuse pulmonary infiltrates ${ }^{14}$.

Most sobering of all from the report presented by Martínez-Martínez, et al was that 3 deaths were attributable to undiagnosed and untreated IFD. Appropriate and timely therapy may decrease mortality for patients with IFD. Prophylactic antifungal agents are clearly warranted in an immunosuppressed population following stem cell transplants ${ }^{16}$ and following solid organ transplants in some centers (as reviewed by Subramanian ${ }^{17}$ ). There are currently no guidelines for the use of prophylactic antifungal agents in the SLE population. Prediction rules and biomarkers, such as serum $(1,3)-\beta-D$-glucan for invasive candida infections, have been developed, and continuing research is aimed at developing early treatment strategies to reduce morbidity and mortality ${ }^{18}$. New prospects are monoclonal antibodies that are currently being developed and tested as vaccines and treatments for IFD ${ }^{6}$.

At present, however, we can only encourage our medical community, particularly rheumatology, infectious disease, and intensive care physicians, to consider IFD in the differential diagnosis of patients with severe SLE who present with features of infection, and limit unnecessary corticosteroid use.

CLAIRE E. BARBER, MD, FRCPC; CHERYL BARNABE, MD, FRCPC, MSC, University of Calgary, Division of Rheumatology, 3280 Hospital Drive NW, Calgary, Alberta T2N 4N6, Canada

Address correspondence to Dr. Barber; E-mail: cehbarbe@ucalgary.ca Dr. Barber is supported by the UCB-CRA-TAS Post-Graduate Rheumatology Fellowship and Alberta Innovates Health Solutions Clinical Fellowship.

\section{REFERENCES}

1. Cervera R, Khamashta MA, Font J, Sebastiani GD, Gil A, Lavilla P, et al. Morbidity and mortality in systemic lupus erythematosus during a 10-year period: A comparison of early and late manifestations in a cohort of 1,000 patients. Medicine (Baltimore) 2003;825:299-308.

2. Goldblatt F, Chambers S, Rahman A, Isenberg DA. Serious infections in British patients with systemic lupus erythematosus: Hospitalisations and mortality. Lupus 2009;18:682-9.

3. Garred P, Madsen HO, Halberg P, Petersen J, Kronborg G, Svejgaard A, et al. Mannose-binding lectin polymorphisms and susceptibility to infection in systemic lupus erythematosus. Arthritis Rheum 1999;42:2145-52.

4. Yong PF, Aslam L, Karim MY, Khamashta MA. Management of hypogammaglobulinaemia occurring in patients with systemic lupus erythematosus. Rheumatology 2008;47:1400-5.

5. Dias AM, do Couto MC, Duarte CC, Ines LP, Malcata AB. White blood cell count abnormalities and infections in one-year follow-up of 124 patients with SLE. Ann NY Acad Sci 2009;1173:103-7.

6. Ito JI. T cell immunity and vaccines against invasive fungal diseases. Immunol Invest 2011;40:825-38.

7. Navarro-Zarza JE, Alvarez-Hernandez E, Casasola-Vargas JC, Estrada-Castro E, Burgos-Vargas R. Prevalence of community-acquired and nosocomial infections in hospitalized patients with systemic lupus erythematosus. Lupus 2010;19:43-8.

8. Ascioglu S, Rex JH, de Pauw B, Bennett JE, Bille J, Crokaert F, et al. Defining opportunistic invasive fungal infections in immunocompromised patients with cancer and hematopoietic stem cell transplants: An international consensus. Clin Infect Dis 2002;34:7-14.

9. De Pauw B, Walsh TJ, Donnelly JP, Stevens DA, Edwards JE, Calandra T, et al. Revised definitions of invasive fungal disease from the European Organization for Research and Treatment of Cancer/Invasive Fungal Infections Cooperative Group and the National Institute of Allergy and Infectious Diseases Mycoses Study Group (EORTC/MSG) Consensus Group. Clin Infect Dis 2008;46:1813-21.

10. Martínez-Martínez M, Herrera-Van Oostdam D, Román-Acosta S, Magaña-Aquino M, Baranda-Cándido L, Abud-Mendoza C. Invasive fungal infections in patients with systemic lupus

Personal non-commercial use only. The Journal of Rheumatology Copyright (C) 2012. All rights reserved. 
erythematosus. J Rheumatol 2012;39:1814-8.

11. Chen GL, Chen Y, Zhu CQ, Yang CD, Ye S. Invasive fungal infection in Chinese patients with systemic lupus erythematosus. Clin Rheumatol 2012;31:1087-91.

12. Chen HS, Tsai WP, Leu HS, Ho HH, Liou LB. Invasive fungal infection in systemic lupus erythematosus: An analysis of 15 cases and a literature review. Rheumatology 2007;46:539-44.

13. Gladman DD, Ibanez D, Urowitz MB. Systemic Lupus Erythematosus Disease Activity Index 2000. J Rheumatol 2002;29:288-91.

14. Sieving RR, Kauffman CA, Watanakunakorn C. Deep fungal infection in systemic lupus erythematosus - Three cases reported, literature reviewed. J Rheumatol 1975;2:61-72.

15. Kim HJ, Park YJ, Kim WU, Park SH, Cho CS. Invasive fungal infections in patients with systemic lupus erythematosus:
Experience from affiliated hospitals of Catholic University of Korea. Lupus 2009;18:661-6.

16. Tomblyn M, Chiller T, Einsele H, Gress R, Sepkowitz K, Storek J, et al. Guidelines for preventing infectious complications among hematopoietic cell transplantation recipients: A global perspective. Biol Blood Marrow Transplant 2009;15:1143-238.

17. Subramanian AK. Antimicrobial prophylaxis regimens following transplantation. Curr Opin Infect Dis 2011;24:344-9.

18. Ostrosky-Zeichner L, Kullberg BJ, Bow EJ, Hadley S, Leon C, Nucci M, et al. Early treatment of candidemia in adults: A review. Med Mycol 2011;49:113-20.

J Rheumatol 2012;39:1772-4; doi:10.3899/jrheum.120707 